Supporting Information

\title{
High Performance Bubble Manipulation on Ferrofluid- Infused Laser-Ablated Microstructured Surfaces
}

Suwan Zhu, ${ }^{\dagger}$ Y ucheng Bian,, Tao Wu,${ }^{\S}$ Chao Chen,${ }^{\dagger}$ Y Yunlong Jiao, ${ }^{\dagger}$ Zhiwu Jiang, ${ }^{\S}$ Zhouchen

Huang, ${ }^{\dagger}$ Erqiang Li,$\S$ Jiawen Li ${ }^{*}, \dagger$ Jiaru Chu, ${ }^{\dagger}$ Yanlei Hu, ${ }^{*, \dagger}$ Dong Wu, ${ }^{*}, \dagger$ and Lei Jiang\|

†CAS Key Laboratory of Mechanical Behavior and Design of Materials, Key Laboratory of Precision Scientific Instrumentation of Anhui Higher Education Institutes, Department of Precision Machinery and Precision Instrumentation, University of Science and Technology of China, Hefei 230026, China.

\$School of Microelectronics, University of Science and Technology of China, Hefei 230026, China.

$\S$ Department of Modern Mechanics, University of Science and Technology of China, Hefei 230026, China.

\|Key Laboratory of Bio-inspired Materials and Interfacial Science, Technical Institute of Physics and Chemistry, Chinese Academy of Sciences, Beijing 100190, China

KEYWORDS: gas bubbles, ferrofluid, femtosecond laser, slippery surfaces, bubble manipulation 


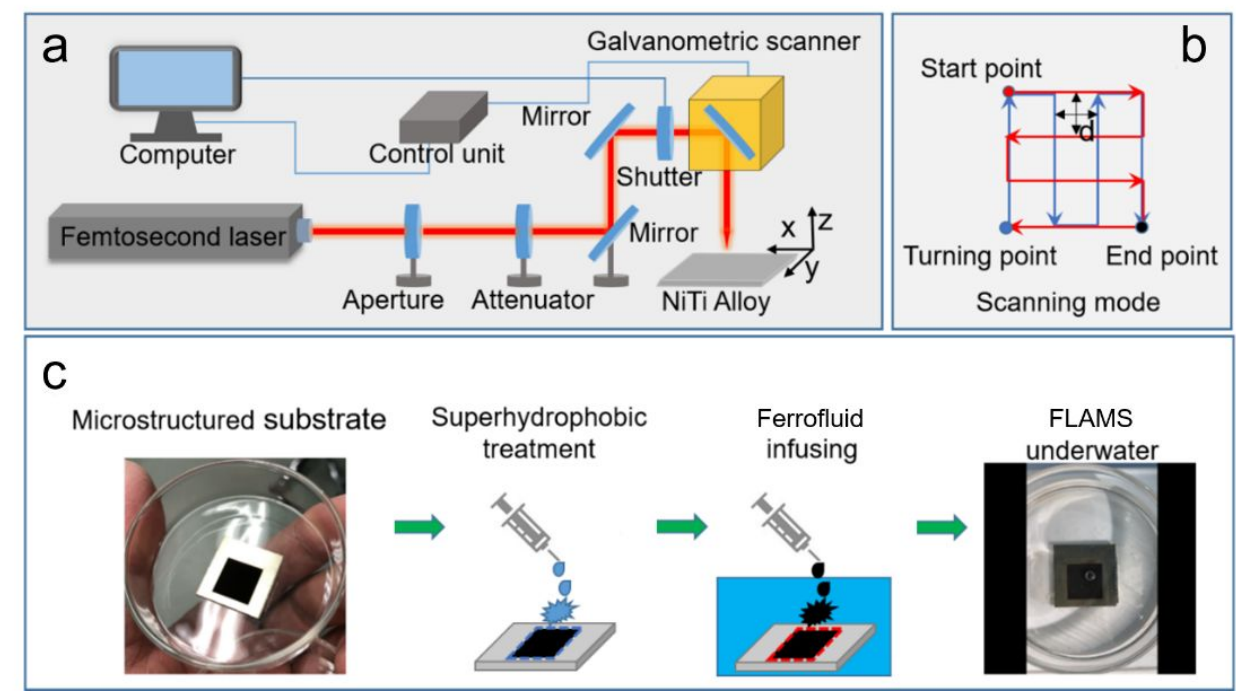

Figure S1. (a) Optical pathway for femtosecond-laser ablation system . (b) Scanning path of laser ablation on the samples. The NiTi alloy sheet is microstructured by vertically crossed lineby-line laser ablation. (c) The as-prepared microstructured substrate is placed under water and then infused with a certain amount of octane-based ferrofluid by a microsyringe, to form the FLAMS.

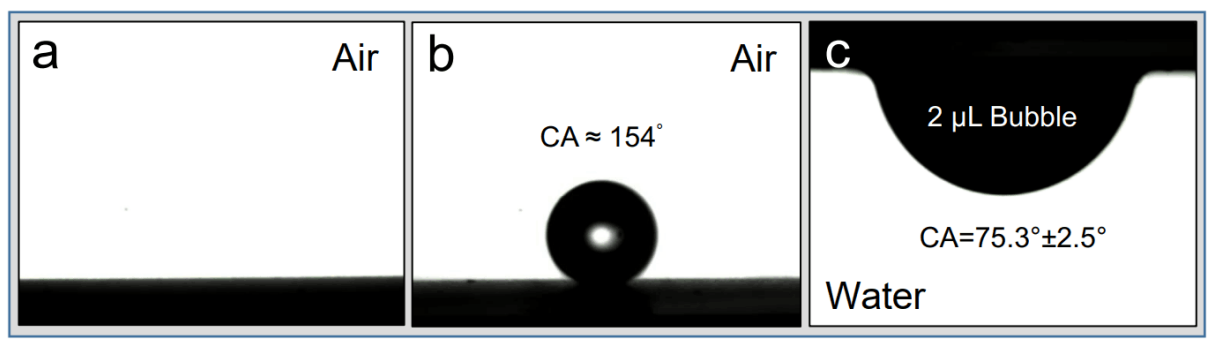

Figure S2. (a) Water contact angle (WCA) is measured to be $\sim 0^{\circ}$ on laser-ablated substrate with super-hydrophilicity. (b) After superhydrophobic treatment, the surface shows a WCA of $\sim 154^{\circ}$. (c) Underwater FLAMS shows a bubble contact angle (BCA) of $75.3^{\circ} \pm 2.5^{\circ}$ with aerophilicity. 


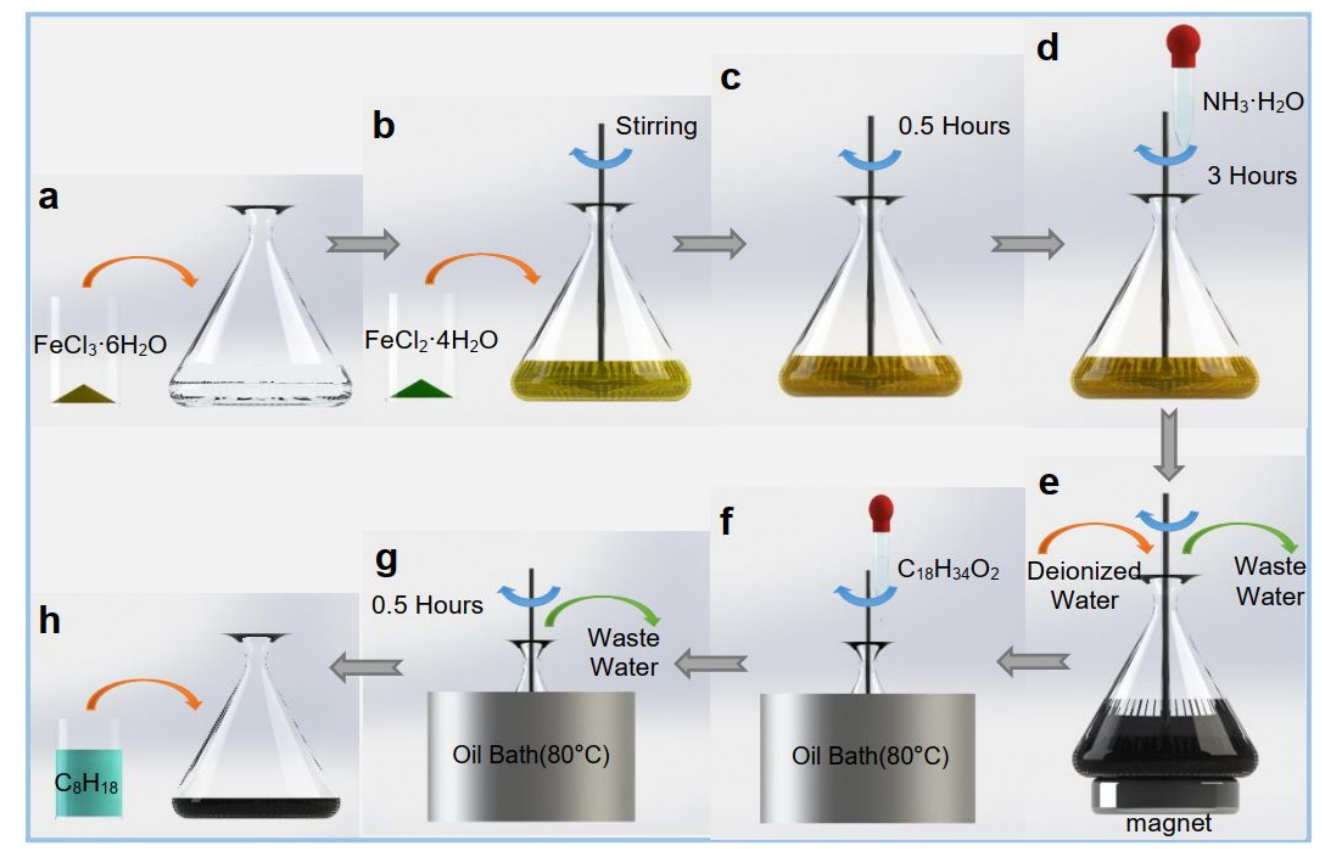

Figure S3. Synthesis of ferrofluid. (a) $\mathrm{FeCl}_{3} \cdot 6 \mathrm{H}_{2} \mathrm{O}(5.4 \mathrm{~g}, 20 \mathrm{mmol})$ and (b) $\mathrm{FeCl}_{2} \cdot 4 \mathrm{H}_{2} \mathrm{O}(2.0$ g, $10 \mathrm{mmol}$ ) are added to a conical flask containing $200 \mathrm{~mL}$ DI water respectively and then (c) stirred for $30 \mathrm{~min}$. (d) $\mathrm{NH}_{3} \cdot 4 \mathrm{H}_{2} \mathrm{O}(25 \mathrm{wt} \%, 7 \mathrm{~mL})$ is added dropwise to the mixture under vigorous stirring, forming $\mathrm{Fe}_{3} \mathrm{O}_{4}$ nanocrystals in weak alkaline solution. After stirring for 3 hours, the resulting precipitate is isolated from the mixture with a strong magnet, washed with deionized water for three times. (e) Oleic acid (1.22 g, $4.3 \mathrm{mmol})$ is added dropwise to $\mathrm{Fe}_{3} \mathrm{O}_{4} /$ water suspension at $80{ }^{\circ} \mathrm{C}$ under (f) nitrogen protection and $(\mathrm{g})$ continuous stirring for 30 minutes before pouring out the waste water. (h) Finally, the surface-modified $\mathrm{Fe}_{3} \mathrm{O}_{4}$ nanocrystals are uniformly dispersed in $\mathrm{C}_{8} \mathrm{H}_{18}$ and ready for use. 


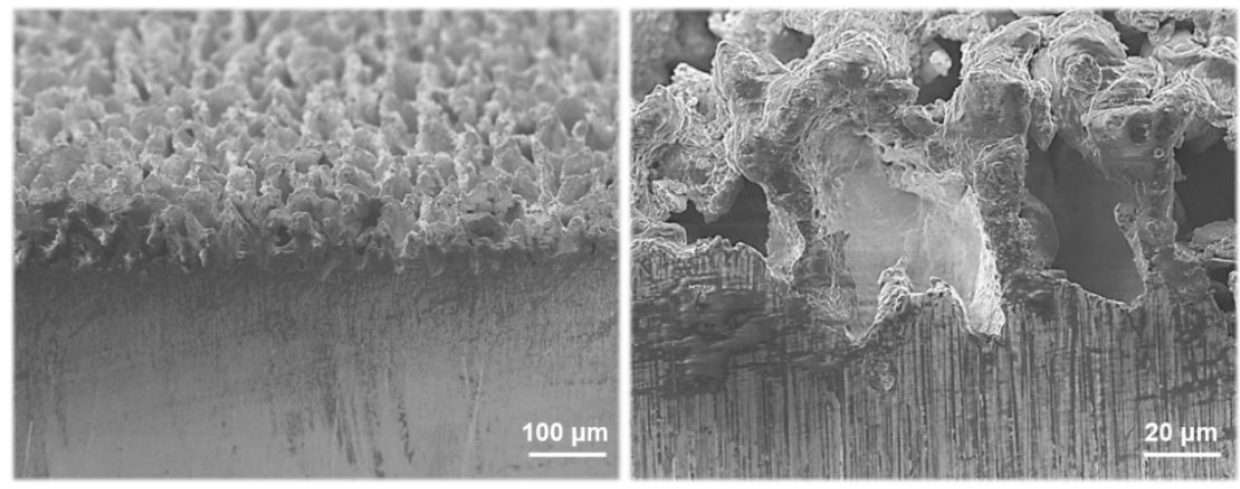

Figure S4. Cross-sectional structures of Ni-Ti alloy substrate observed by SEM at different magnification, in which the average depth of the groove structure is measured to be $\sim 60 \mu \mathrm{m}$.

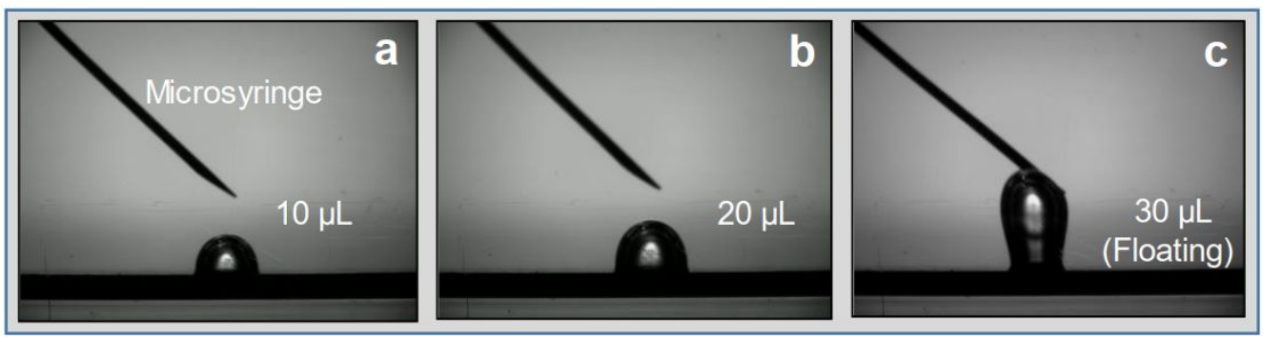

Figure S5. (a) A $10 \mu \mathrm{L}$ bubble on the FLAMS. (b) A $20 \mu \mathrm{L}$ bubble on the FLAMS. (c) Once bubble volume increases to $30 \mu \mathrm{L}$, the bubble detaches from the FLAMS and rises upward in water. 


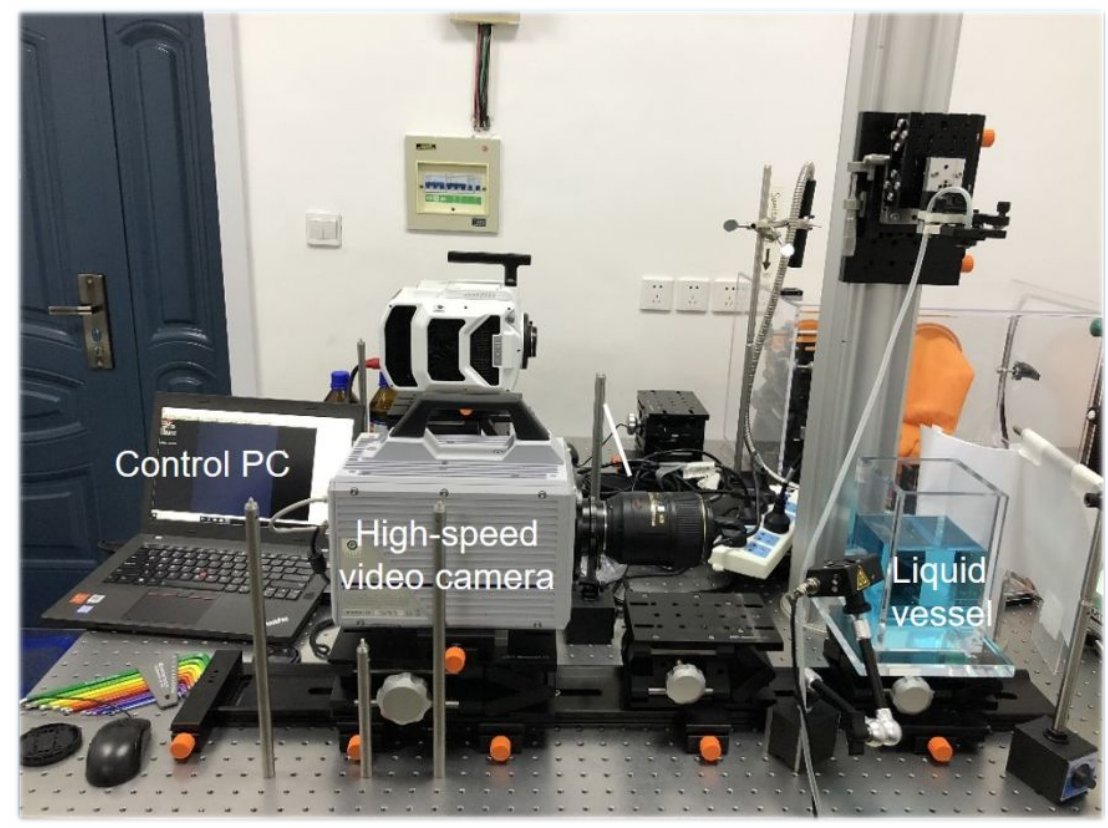

Figure S6. High-speed image acquisition system consists of high-speed video cameras, an acrylic liquid vessel and a control PC. The FLAMS platform is fixed on the bottom surface of the acrylic vessel. The dynamic interaction between bubble and FLAMS-water interface is investigated by high-speed video camera at a typical rate of 10, $000 \mathrm{fps}$.

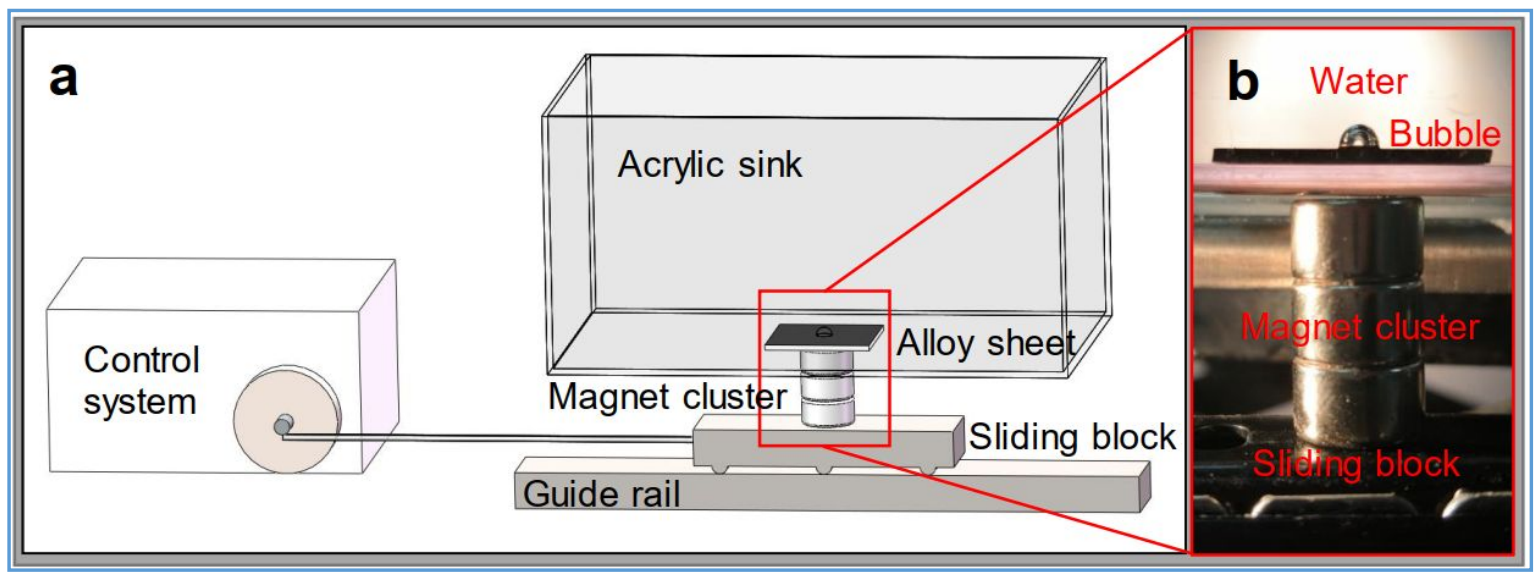

Figure S7. (a) Purpose-built bubble driving system, including control system, sliding block, guide rail, magnet cluster and acrylic sink. The moving velocity of the magnet cluster can be tuned by different current that applied on electromotor. (b) The magnified photograph of the sliding block and FLAMS-attached bubble. 

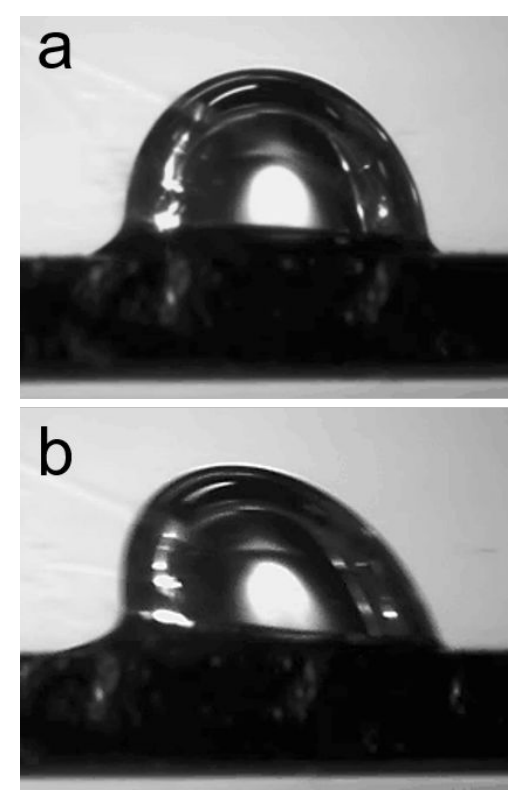

Figure S8. Meniscus deformation during the bubble motion. (a) $V_{\mathrm{d}}=5 \mathrm{~mm} / \mathrm{s}$, (b) $V_{\mathrm{d}}=35 \mathrm{~mm} / \mathrm{s}$. The bubble motion is driven by the deformation and displacement of the ferrofluid meniscus induced by the movement of bottom magnetic field. Consequently, response time is needed which would cause the hysteresis between bubble motion and the magnetic field movement. While the driving velocity $V_{\mathrm{d}}$ of the magnetic source elevated (from $5 \mathrm{~mm} / \mathrm{s}$ to 35 $\mathrm{mm} / \mathrm{s}$ ), the hysteresis would intensify, as a result, the deviation distance of the bubble from the center of the magnetic source would increase, and the ferrofluid accumulated in front of the bubble movement direction would also increase (Figure S8). Meanwhile, the bubble needs to overcome more viscous resistance to follow the magnetic source. Once the distance between the bubble and the magnetic source exceeded the effective range of the magnetic field, the bubble would stop moving. The increase of the driving velocity $V_{\mathrm{d}}$ will accelerate the separation of the bubble from the magnetic field, resulting in a shorter movement distance. The bubble motion is also influenced by the viscous friction stemming from the relative motion with the ambient fluid. At low Reynolds numbers, the drag force $f$ of an ideal non-deformable spherical bubble moving under water follows the Hardamard-Rubczynski formula, ${ }^{[1,2]}$

$$
f=4 \pi \mu R U
$$

where $\mu, R$, and $U$ are the dynamic viscosity of the water, the radius of bubble, and the velocity of bubbles, respectively. The sectional area of the bubble perpendicular to the movement direction would increase as the elevation of bubble volume, resulting in the enlargement of the viscous resistance caused by the surrounding fluid. Hence, the bubble movement would be terminated earlier. 


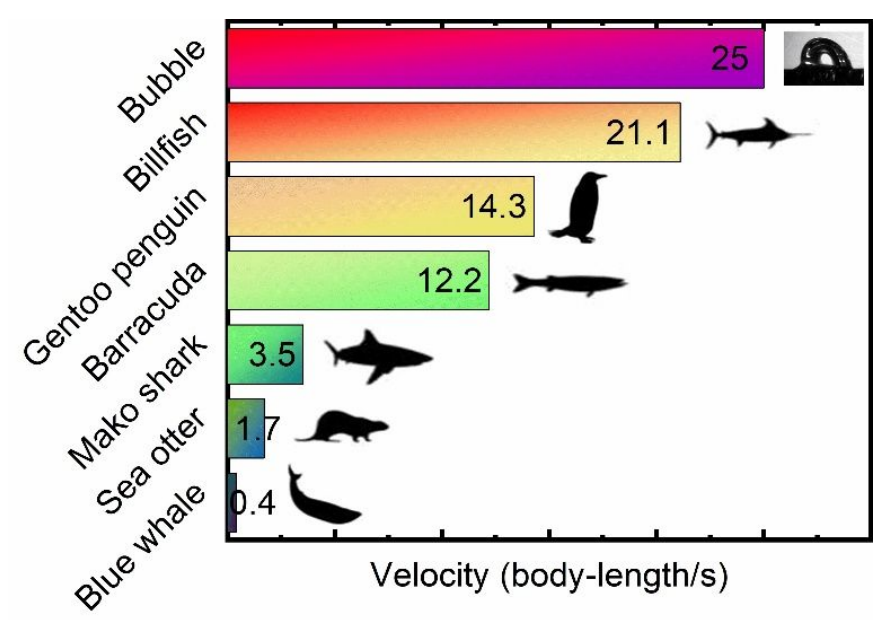

Figure S9. Comparison of the bubble motion efficiency with various marine lives. A dimensionless number $V_{\mathrm{bl}}$ is defined as the travelling distance per second relative to the bodylength. Typical gas bubble $(<2 \mu \mathrm{L})$ could reach up to a $V_{\mathrm{bl}}$ of 25 , which exceeds the maximum burst speed record (21.1 body-length/s) for billfishes considered to be among the fastest swimmers in the oceans.

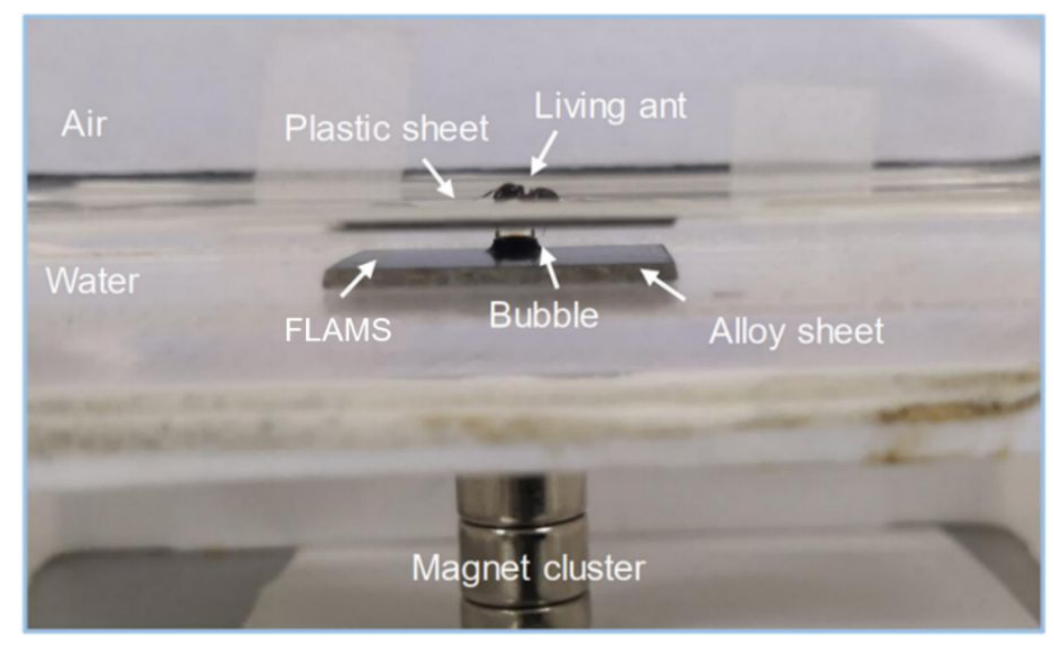

Figure S10. Proof-of-concept "bubble mini-robot" (BMR) for controlling a plastic sheet with a living carpenter on its surface. The dome of the FLAMS-attached BMR is physically connected with the lower surface of the plastic sheet. 


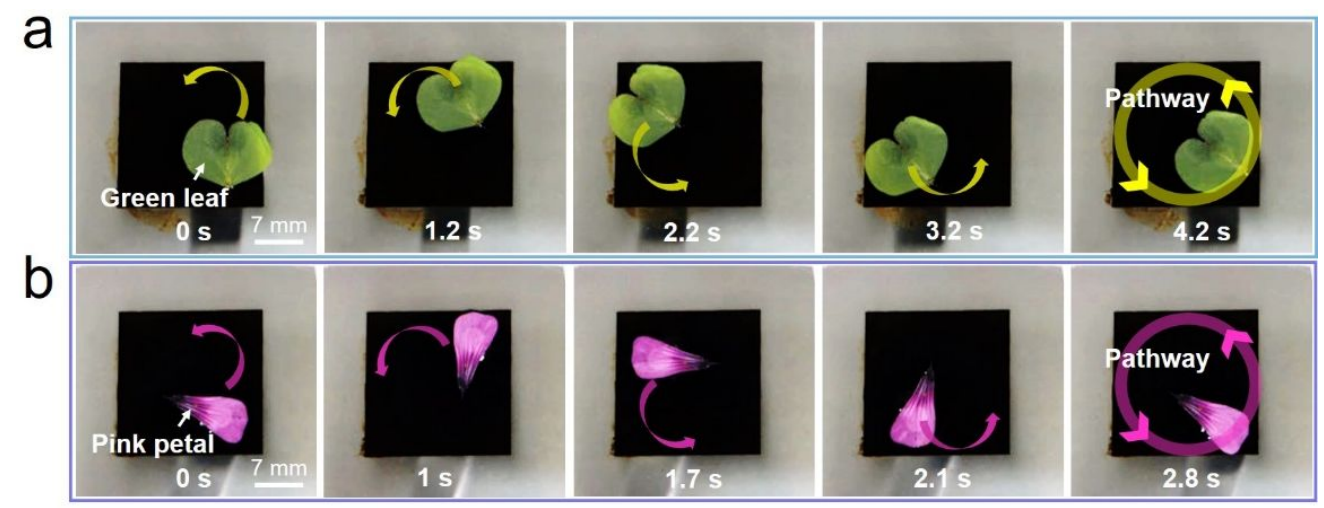

Figure S11. Matter transport of (a) a $10 \mathrm{mg}$ green leaf and (b) a $3 \mathrm{mg}$ pink petal on the airwater interface by a BMR. The green leaf and the pink petal are guided by the BMR to complete the circular motion within $\sim 4.2 \mathrm{~s}$ and $\sim 2.8 \mathrm{~s}$ respectively, demonstrating the high towing capacity of transporting a much heavier object ( $>500$ own mass) for BMR.

\section{Supporting References}

[1] A. Tomiyama, I. Kataoka, I. Zun, and T. Sakaguchi. JSME Int. J., Ser. B 41(2), 472-479 (1998).

[2] I. U. Vakarelski, J. O. Marston, D. Y. C. Chan, and S. T. Thoroddsen. Phys. Rev. Lett. 106(21), 214501 (2011). 